surgery $(\mathrm{p}=0.33)$. This study confirmed a well known data in other surgical specialties, including ours own, that previous abdominal surgery does not increase the morbidity of laparoscopic procedures. The pivotal issue of surgical techniques always point out to the surgeons' experience with particular surgical technique.

Dr. Fernando J. Kim

Chief of Urology, Denver Health Med. Ctr. Associate Professor, Univ. Colorado Health Sci. Ctr. Director of Minimally Invasive Urol. Oncology, UCHSC

Denver, Colorado, USA

E-mail:fernando.kim@dhha.org

\title{
IMAGING
}

doi: $10.1590 / S 1677-55382010000600020$

\section{Ultrasound assessment of intravesical prostatic protrusion and detrusor wall thickness--new standards for noninvasive bladder outlet obstruction diagnosis?}

Franco G, De Nunzio C, Leonardo C, Tubaro A, Ciccariello M, De Dominicis C, Miano L, Laurenti C

Department of Urology, La Sapienza University, Rome, Italy

J Urol. 2010; 183: 2270-4

Purpose: We evaluated the accuracy of detrusor wall thickness and intravesical prostatic protrusion, and the association of each test to diagnose bladder prostatic obstruction in patients with lower urinary tract symptoms. Materials and Methods: We enrolled in the study 100 consecutive patients with lower urinary tract symptoms due to benign prostatic hyperplasia. Baseline parameters were International Prostate Symptom Score, prostate volume, urinary flow rate, intravesical prostatic protrusion, detrusor wall thickness, Schaefer obstruction class, minimal urethral opening pressure and the urethral resistance algorithm bladder outlet obstruction index. A ROC curve was produced to calculate AUC and evaluate the diagnostic performance of intravesical prostatic protrusion, detrusor wall thickness and prostate volume for bladder prostatic obstruction.

Results: We noted a highly significant correlation between intravesical prostatic protrusion and the bladder outlet obstruction index (Spearman's rho $=0.49, \mathrm{p}=0.001$ ), and Schaefer obstruction class (Spearman's rho = $0.51, \mathrm{p}=0.001)$. A highly significant correlation was also observed for detrusor wall thickness and the bladder outlet obstruction index (Spearman's rho $=0.57, \mathrm{p}=0.001$ ), detrusor wall thickness and Schaefer obstruction class (Spearman's rho $=0.432, \mathrm{p}=0.02)$. On multivariate analysis intravesical prostatic protrusion and detrusor wall thickness were the only parameters associated with bladder prostatic obstruction $(\mathrm{p}=0.015)$. The AUC for intravesical prostatic protrusion was 0.835 (95\% CI 0.756-0.915) and for detrusor wall thickness it was 0.845 (95\% CI 0.78-0.91). The association of intravesical prostatic protrusion and detrusor wall thickness produced the best diagnostic accuracy $(87 \%)$ when the 2 tests were done consecutively.

Conclusions: Suprapubic ultrasound of detrusor wall thickness and intravesical prostatic protrusion is a simple, noninvasive, accurate system to assess bladder prostatic obstruction in patients with lower urinary tract symptoms due to benign prostatic hyperplasia.

\section{Editorial Comment}

Recently attempts have been made to provide noninvasive tests for the assessment of bladder outlet obstruction (BOO). Since prostate volume and post void residual urine are not accurate parameters in predict- 
ing BOO, the positive predict value of others sonographic measurements such detrusor wall thickness (DWT), bladder weight and intravesical prostatic protrusion (IPP) has been investigated. In this study the authors evaluated a group of 100 patients with lower urinary tract symptoms due to benign prostatic hyperplasia. They found that the AUC for IPP was 0.835 with a cutoff of $12 \mathrm{~mm}$ having 0.65 sensitivity and 0.77 specificity and $88 \%$ positive predictive value. The AUC for DWT was 0.845 and at a $6 \mathrm{~mm}$ cutoff they noted 0.73 sensitivity and 0.82 specificity. Patients with 1 of the 2 tests positive (IPP $12 \mathrm{~mm}$ or more, or DWT $7 \mathrm{~mm}$ or more) have an approximately $90 \%$ chance of bladder prostatic obstruction on pressure flow study. In the evaluation of IPP, the authors excluded men with a median lobe, which may cause ball valve type of obstruction and can be easily determined by suprapubic US in the sagittal plane. Special attention was given to the role of protrusion of lateral lobes into the bladder. Radiologists and urologists, who perform urological ultrasound, should be aware of the technical aspects of how to obtain these measurements. The estimative of DWT, is yet the only one of these two parameters which has been recommended by the "American College of Radiology 2008 - Appropriateness Criteria", requires adequate maximum bladder volume, adequate place of the transducer for bladder wall measurement and optimized ultrasound transducer frequency.

\author{
Dr. Adilson Prando \\ Head, Department of Radiology and \\ Diagnostic Imaging, Vera Cruz Hospital \\ Campinas, São Paulo, Brazil \\ E-mail: adilson.prando@gmail.com
}

doi: $10.1590 / S 1677-55382010000600021$

\title{
Renal cell carcinoma: diffusion-weighted MR imaging for subtype differentiation at 3.0 T
}

Wang H, Cheng L, Zhang X, Wang D, Guo A, Gao Y, Ye H

Department of Radiology, PLA General Hospital, Beijing, China

Radiology. 2010; 257: 135-43

Purpose: To assess the usefulness of apparent diffusion coefficients (ADCs) for characterizing renal cell carcinoma (RCC) subtypes at 3.0 T.

Materials and Methods: The Institutional Review Board approved this retrospective study, and informed consent was waived. Eighty-three patients underwent diffusion-weighted (DW) magnetic resonance (MR) imaging of 85 renal masses. In each patient, precontrast single-shot spin-echo echo-planar DW imaging was performed with $\mathrm{b}$ values of 0 and 500 and 0 and $800 \mathrm{sec} / \mathrm{mm}(2)$ by using a 3.0-T MR imaging system. Differences in ADCs between the RCC lesions and uninvolved renal parenchyma were tested by using a paired-samples $t$ test. One-way analysis of variance was used to compare ADCs of the various RCC subtypes. Receiver operating characteristic (ROC) curve analysis was used to test the ability of ADCs in differentiating clear cell from non-clear cell RCCs.

Results: Pathologic diagnoses of the 85 tumors (median diameter, $4.4 \mathrm{~cm}$ ) in the 83 patients $(54 \mathrm{men}, 29$ women; age range, 23-75 years; mean age, 49.4 years) were clear cell RCC for 49 tumors, papillary RCC for 22 tumors, and chromophobic RCC for 14 tumors. With b values of 0 and $500 \mathrm{sec} / \mathrm{mm}(2)$, clear cell RCCs showed a significantly higher mean ADC $(1.849 \times 10(-3) \mathrm{mm}(2) / \mathrm{sec})$ than papillary $(1.087 \times 10(-3) \mathrm{mm}(2) / \mathrm{sec})$ and chromophobic $(1.307 \times 10(-3) \mathrm{mm}(2) / \mathrm{sec}) \mathrm{RCCs}(\mathrm{P}<.001)$; however, the difference between papillary and chromophobic RCCs was not significant $(\mathrm{P}=.068)$. With b values of 0 and $800 \mathrm{sec} / \mathrm{mm}(2)$, clear cell RCC showed the largest mean ADC $(1.698 \times 10(-3) \mathrm{mm}(2) / \mathrm{sec})$ of the three subtypes, and the difference between each pair of 
subtypes was significant $(\mathrm{P}<.001)$. ADCs obtained with $\mathrm{b}$ values of 0 and $800 \mathrm{sec} / \mathrm{mm}(2)$ were more effective for distinguishing clear cell from non-clear cell RCC (area under the ROC curve, 0.973): A threshold value of $1.281 \times 10(-3) \mathrm{mm}(2) / \mathrm{sec}$ permitted distinction with high sensitivity $(95.9 \%)$ and specificity $(94.4 \%)$.

Conclusion: DW imaging with $\mathrm{b}$ values of 0 and $800 \mathrm{sec} / \mathrm{mm}(2)$ allows sensitive and specific differentiation of clear cell, papillary, and chromophobic RCCs, suggesting that DW imaging may be useful in the preoperative characterization of RCC.

\section{Editorial Comment}

Diffusion-weighted imaging (DWI) is a magnetic resonance technique where the contrast between tissues derives from the regional differences in the mobility of water molecules. The diffusion data can be presented as signal intensity on pure DWI or as an image map of the apparent diffusion coefficient (ADC). Calculation of the ADC requires 2 or more acquisitions with different diffusion weightings ("b values "). A low ADC corresponds to high signal intensity in pure DWI (restricted diffusion usually found in more compact tissues), and a high ADC to low signal intensity on pure DWI (found in less compact tissue). The authors of this study evaluated with DWI 85 tumors (49 clear cells RCC, 22 papillary RCC and 14 chromophobic RCC. ADCs obtained with b values of 0 and $800 \mathrm{sec} / \mathrm{mm}^{2}$, were more effective for distinguishing clear cell from non-clear cell RCC (area under the ROC curve, 0.973), and for distinguishing RCC sub types.

DWI has also been shown to be useful to distinguish benign from malignant renal tumors (1). Statistically significant differences exist between the ADC values of normal kidney, renal carcinomas, renal angiomyolipomas and renal cysts when the " $b$ value " is the same. In our opinion however further studies are necessary to confirm the reported higher accuracy in distinguishing benign from malignant renal tumors and in distinguishing RCC subtypes. Until now, there is no consensus regarding which are the best " $\mathrm{b}$ values " for renal masses characterization. Do we need just a pair of b values (example: 0 and $800 \mathrm{sec} / \mathrm{mm}^{2}$ ) or do we need a multi-b-values technique (example: b values of $0,50,100,300,500,800,1000$, etc) in order to obtain a discriminatory characteristic curve of ADC values?. Another point to consider is that in the majority of situations, radiologists use only visual assessment of DWI; in other words normal or benign, less compact tissues, usually present as an area with lower signal intensity on DWI and with higher ADC values. Contrary, tumoral compact tissue, due its restricted diffusion, appears as an area with higher signal intensity on DWI and lower ADC values. However in our preliminary experience we have been seen benign angiomyolipoma with lower $\mathrm{ADC}$ values than RCC.

\section{Reference}

1. Zhang Y, Sun Xingwang, Quan G, Qiang Y, Li C: Value of apparent diffusion coefficient (ADC) of diffusion weighted magnetic resonance imaging in common renal disease diagnosis. Journal of Nanjing Medical University 2008; 22: $362-365$.

Dr. Adilson Prando Head, Department of Radiology and Diagnostic Imaging, Vera Cruz Hospital Campinas, São Paulo, Brazil E-mail: adilson.prando@gmail.com 